\title{
Simplifying strong electronic correlations in uranium: Localized uranium heavy-fermion $\mathrm{UM}_{2} \mathrm{Zn}_{20}(M=\mathrm{Co}, \mathrm{Rh})$ compounds
}

\author{
E. D. Bauer, ${ }^{1}$ C. Wang,,${ }^{1} *$ V. R. Fanelli, ${ }^{1, *}$ J. M. Lawrence, ${ }^{2}$ E. A. Goremychkin, ${ }^{3}$ N. R. de Souza, ${ }^{3}$ F. Ronning, ${ }^{1}$ \\ J. D. Thompson, ${ }^{1}$ A. V. Silhanek, ${ }^{1}$ V. Vildosola, ${ }^{4}$ A. M. Lobos,${ }^{5}$ A. A. Aligia, ${ }^{5}$ S. Bobev, ${ }^{6}$ and J. L. Sarrao ${ }^{1}$ \\ ${ }^{1}$ Los Alamos National Laboratory, Los Alamos, New Mexico 87545, USA \\ ${ }^{2}$ University of California, Irvine, Irvine, California 92697, USA \\ ${ }^{3}$ Argonne National Laboratory, Argonne, Illinois 60439, USA \\ ${ }^{4}$ Departamento de Física, Centro Atómico Constituyentes, Comisión Nacional de Energía Atómica, 1429 Buenos Aires, Argentina \\ ${ }^{5}$ Centro Atómico Bariloche and Instituto Balseiro, Comisión Nacional de Energía Atómica, 8400 Bariloche, Argentina \\ ${ }^{6}$ Department of Chemistry and Biochemistry, University of Delaware, Newark, Delaware 19716, USA
}

(Received 12 June 2008; published 29 September 2008)

\begin{abstract}
The physical properties including magnetic susceptibility, magnetization, specific heat, and dynamic susceptibility $\chi^{\prime \prime}(E)$ are reported for single crystals of the cubic $\mathrm{UM}_{2} \mathrm{Zn}_{20}(\mathrm{M}=\mathrm{Co}, \mathrm{Rh})$ materials. Maxima in the thermodynamic data at $T_{\max } \sim 10 \mathrm{~K}$ for both compounds and a broad peak in $\chi^{\prime \prime}(E)$ at $5 \mathrm{~K}$ in $\mathrm{UCo}_{2} \mathrm{Zn}_{20}$ of width $\Gamma=5 \mathrm{meV}$ indicate a heavy-fermion state characterized by a Kondo temperature $T_{K} \sim 20-30 \mathrm{~K}$ arising from weak hybridization of $f$ - and conduction-electron states. Anderson impurity model fits to the data in the Kondo limit including crystalline electric-field effects corroborate an ionic-like uranium electronic configuration in $\mathrm{UM}_{2} \mathrm{Zn}_{20}$.
\end{abstract}

DOI: $10.1103 /$ PhysRevB.78.115120

PACS number(s): 71.27.+a, 72.15.Qm

\section{INTRODUCTION}

The essential physics of strongly correlated electron materials is deceptively simple: strong on-site Coulomb repulsion of $d$ or $f$ electrons promote localization while orbital overlap with neighboring ligands leads to $d$ - $(f$-) electron itineracy. While the delicate balance between these two competing tendencies generate novel phenomena in both $d$-electron systems (e.g., cuprates ${ }^{1}$ and ruthenates ${ }^{2}$ ) and $f$-electron heavy-fermion compounds, ${ }^{3}$ the complexity resulting from such competition is generally intractable. This insolubility is particularly evident in the actinide heavyfermion metals, ${ }^{4}$ where even the question of whether to treat the $5 f$ electrons as localized or itinerant has not been answered because their orbitals are spatially extended and dispersive; hence, few exemplary systems have been found on the simpler localized side of the spectrum. As discussed herein, the new strongly correlated $\mathrm{UM}_{2} \mathrm{Zn}_{20}(\mathrm{M}=\mathrm{Co}, \mathrm{Rh})$ compounds represent a particularly simple limit of this complexity; their crystal chemistry leads to $4 f$-like behavior that allows straightforward insight into their underlying physics.

Uranium-based heavy-fermion (HF) metals share characteristic behavior with rare-earth-based $\mathrm{HF}$ materials, ${ }^{4-6}$ namely that their low-temperature Pauli paramagnetic susceptibility $\chi_{0}$ and the linear coefficient of specific heat $\gamma$ $=C / T$ are large, reflecting a strongly renormalized mass of charge carriers that is 100-1000 times the free electron mass. Both $\chi_{0}$ and $\gamma$ scale as $1 / T_{s f}$, where the characteristic spin fluctuation energy $k_{B} T_{s f}$ is small $\left(T_{s f} \sim 1-100 \mathrm{~K}\right)$ and can be observed directly as the energy of a strongly damped peak in the dynamic susceptibility $\chi^{\prime \prime}(E)$, measured through inelastic neutron scattering. ${ }^{6}$ This common scaling behavior obscures an important underlying difference: the rare-earth $4 f$ orbitals are highly localized, hybridizing weakly with the conduction electrons, while the uranium $5 f$ orbitals are spatially extended, forming dispersive $f$ bands with strong hybridization with neighboring orbitals. The local-moment character of rare-earth $\mathrm{HF}$ compounds allows for detailed fits of the specific heat, magnetic susceptibility, and dynamic susceptibility to the Anderson impurity model (AIM).$^{7,8}$ On the other hand, sharp crystalline electric field (CEF) levels, a signature of the local character of the $4 f$ electrons in rare-earth compounds, are almost never observed in uranium-based compounds in which the fluctuations of the $5 f$ charge configuration make it impossible to characterize the $5 f$ wave functions by welldefined valence states. It is more common in this case to view the heavy-fermion behavior as arising from spin fluctuations in the narrow but dispersive $5 f$ bands. Therefore, essential differences (apart from the scaling laws) might be expected between the detailed behaviors of uranium- and rare-earth-based heavy-fermion materials. The discovery ${ }^{9}$ of new U-based heavy-fermion compounds $\mathrm{UCo}_{2} \mathrm{Zn}_{20}$ and $\mathrm{URh}_{2} \mathrm{Zn}_{20}$ provides a counter example to this expectation. The application of the local-moment (Kondo) limit of the Anderson model within the noncrossing approximation (NCA) gives a good account of the $5 f$-derived characteristics of these materials. Thus, these $\mathrm{UM}_{2} \mathrm{Zn}_{20}(\mathrm{M}=\mathrm{Co}, \mathrm{Rh})$ materials are model systems with which to understand strongly correlated physics.

\section{EXPERIMENTAL DETAILS}

Single crystals $\mathrm{AX}_{2} \mathrm{Zn}_{20}(\mathrm{~A}=\mathrm{Th}, \mathrm{U})$ were grown in $\mathrm{Zn}$ flux. ${ }^{10}$ The crystal structure of the $\mathrm{AX}_{2} \mathrm{Zn}_{20}(\mathrm{~A}=\mathrm{Th}, \mathrm{U})$ materials was determined to be the cubic $\mathrm{Mg}_{3} \mathrm{Cr}_{2} \mathrm{Al}_{18}$ type by means of single crystal $\mathrm{x}$-ray diffraction. ${ }^{11}$ Magnetic measurements were performed from 2 to $350 \mathrm{~K}$ in magnetic fields up to $6 \mathrm{~T}$ using a commercial superconducting quantum interference device (SQUID) magnetometer. Specificheat measurements were carried out in a commercial cryostat from 1.8 to $300 \mathrm{~K}$ in magnetic fields up to $9 \mathrm{~T}$ along the [111] direction for $\mathrm{UCo}_{2} \mathrm{Zn}_{20}$ and in a home-built cryostat 


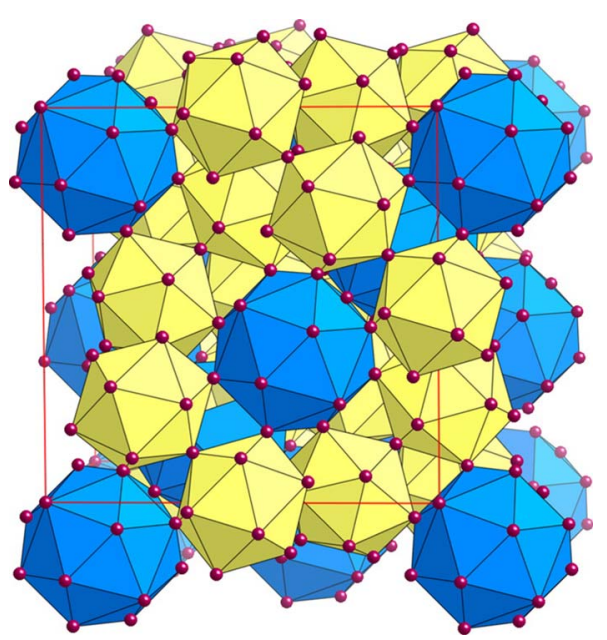

FIG. 1. (Color online) Polyhedral representation of the cubic $\left(\mathrm{Mg}_{3} \mathrm{Cr}_{2} \mathrm{Al}_{18}\right.$ type $)$ structure of $\mathrm{UM}_{2} \mathrm{Zn}_{20}(\mathrm{M}=\mathrm{Co}, \mathrm{Rh})$. The packing of the U-centered $\mathrm{Zn}_{16}$ clusters (blue) and the M-centered $\mathrm{Zn}_{12}$ icosahedra (yellow) is emphasized.

from 1.5 to $300 \mathrm{~K}$ in magnetic fields up to $15 \mathrm{~T}$ along the [110] direction for $\mathrm{URh}_{2} \mathrm{Zn}_{20}$. Inelastic neutron scattering was performed at low temperature (5-6 K) on 20-g powder samples of $\mathrm{UCo}_{2} \mathrm{Zn}_{20}$ and $\mathrm{ThCo}_{2} \mathrm{Zn}_{20}$ on the high energy chopper spectrometer (HET) at ISIS. To obtain an adequate range of energy transfer $2<\Delta E<90 \mathrm{meV}$, three incident energies $\left(E_{i}=23,60\right.$, and $\left.100 \mathrm{meV}\right)$ were used. The absolute cross-section was established by normalizing to vanadium. Measurements at lower energy transfer $(\Delta E<2 \mathrm{meV})$ were carried out on the quasi-elastic neutron spectrometer (QENS) at IPNS. The QENS data have been corrected for absorption, which is significant for total energy (energy transfer plus final energy) below $10 \mathrm{meV}$, but have not been normalized for the absolute cross section. For both experiments, the data for $\mathrm{ThCo}_{2} \mathrm{Zn}_{20}$ have been directly subtracted from the $\mathrm{UCo}_{2} \mathrm{Zn}_{20}$ data to obtain the magnetic scattering function $S_{\text {mag }}(\Delta E)$. This method is based on the observation that the total cross section and absorption cross sections of the two materials are essentially identical, so that direct subtraction removes both the nonmagnetic scattering (assumed equal in the two compounds) and the background (empty holder) scattering. In performing this subtraction, it was found that the phonon peaks at $6.96 \mathrm{meV}$ in $\mathrm{ThCo}_{2} \mathrm{Zn}_{20}$ is shifted to $8.25 \mathrm{meV}$ in $\mathrm{UCo}_{2} \mathrm{Zn}_{20}$. This shift undoubtedly reflects the $5 f$ contribution to the bonding, which increases the phonon energy. This shift was accounted for by fitting the phonon peak to a Gaussian, but shifting its peak energy appropriately when subtracting the Th compound data.

\section{RESULTS AND DISCUSSION}

The $\mathrm{UM}_{2} \mathrm{Zn}_{20}(\mathrm{M}=\mathrm{Co}, \mathrm{Rh})$ compounds crystallize in a cubic structure in which the $\mathrm{U}$ coordination environment comprised exclusively of $\mathrm{Zn}$ atoms is nearly spherical (Fig. 1). In this structure, ${ }^{12}$ each $U$ atom is surrounded by a $Z_{16}$ cluster and each $\mathrm{M}$ atom by a $\mathrm{Zn}_{12}$ cluster, which, given a filled $3 d^{10}$ configuration of $\mathrm{Zn}$, should lead to weak hybrid-

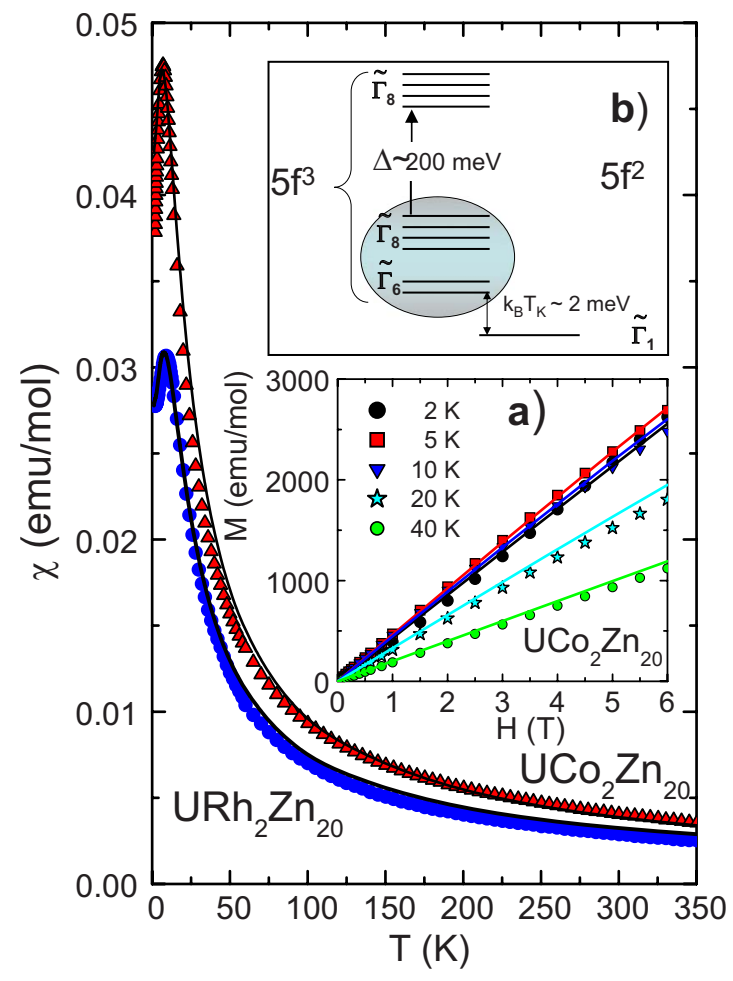

FIG. 2. (Color online) Magnetic susceptibility $\chi(T)$ of $\mathrm{UCo}_{2} \mathrm{Zn}_{20}$ (red triangles) and $\mathrm{URh}_{2} \mathrm{Zn}_{20}$ (blue circles) measured in a magnetic field $H=0.1$ T. (a) Magnetization $M$ vs $H$ between 2 and $40 \mathrm{~K}$ of $\mathrm{UCo}_{2} \mathrm{Zn}_{20}$. (b) CEF energy scheme of $\mathrm{UM}_{2} \mathrm{Zn}_{20}$ $(\mathrm{M}=\mathrm{Co}, \mathrm{Rh})$ discussed in the text. AIM fits (solid lines) to $M(H)$ [(panel (a)] and $\chi(T)$ of $\mathrm{UCo}_{2} \mathrm{Zn}_{20}$ and $\mathrm{URh}_{2} \mathrm{Zn}_{20}$ discussed in the text $\left[M(H)\right.$ fits of $\mathrm{URh}_{2} \mathrm{Zn}_{20}$ similar to (a)].

ization between the $\mathrm{U} 5 f$ and ligand electrons of the $\mathrm{M}$ atoms. It is this special crystal chemistry that simplifies the $5 f$ physics of these materials and controls the physical properties of $\mathrm{UCo}_{2} \mathrm{Zn}_{20}$ and $\mathrm{URh}_{2} \mathrm{Zn}_{20}$ that are summarized in Figs. 2 and $3 .{ }^{9}$ The magnetic susceptibility $\chi(T)$ of the two compounds, displayed in Fig. 2, is typical of heavy-fermion systems with a small characteristic energy scale $\left(T_{K} \sim 30 \mathrm{~K}\right)$, proportional to the temperature of the maximum in $\chi(T)$ at $T_{\max }^{\chi} \sim 7 \mathrm{~K}(9 \mathrm{~K})$ for $\mathrm{UCo}_{2} \mathrm{Zn}_{20}\left(\mathrm{URh}_{2} \mathrm{Zn}_{20}\right)$. The CurieWeiss behavior above $200 \mathrm{~K}$ indicates a localized $5 f$ electronic configuration and the (negative) Curie-Weiss temperature $\theta_{C W}=-65 \mathrm{~K}(-48 \mathrm{~K})$ for $\mathrm{UCo}_{2} \mathrm{Zn}_{20}\left(\mathrm{URh}_{2} \mathrm{Zn}_{20}\right)$ is also consistent with $T_{K}=20-30 \mathrm{~K}\left(\sim \theta_{C W} / 4\right)$. The specific heat, plotted as $C / T$ vs $T$, of $\mathrm{UM}_{2} \mathrm{Zn}_{20}(\mathrm{M}=\mathrm{Co}, \mathrm{Rh})$ is shown in Fig. 3(a) and is enhanced below $10 \mathrm{~K}$ in both compounds. Subtraction of the specific heat of the nonmagnetic isostructural $\mathrm{ThM}_{2} \mathrm{Zn}_{20}(\mathrm{M}=\mathrm{Co}, \mathrm{Rh})$ reveals a broad maximum in the $5 f$ contribution $\Delta C / T$ at $T_{\max }^{C} \sim 4 \mathrm{~K}$ and $6 \mathrm{~K}$ [inset of Fig. 3(a)] for $\mathrm{M}=\mathrm{Co}, \mathrm{Rh}$, respectively. The extrapolated zero-temperature Sommerfeld coefficient is $\gamma \simeq 450$ (300) $\mathrm{mJ} / \mathrm{mol} \mathrm{K}^{2}$ for $\mathrm{M}=\mathrm{Co}(\mathrm{Rh})$. These properties, including the magnetization $M(H)$ [Fig. 2(a)], are reminiscent of uranium magnetic "impurities" embedded in a metallic host described by the Anderson impurity model for a localized $f$ moment with angular momentum $J>1$, where maxima in $C(T)$ and $\chi(T)$ are predicted. ${ }^{13}$ These measurements permit an estimate of the Wilson ratio $\mathcal{R}_{W}=\left(\pi^{2} R / 3 C_{J}\right)\left(\chi_{0} / \gamma\right)$, 


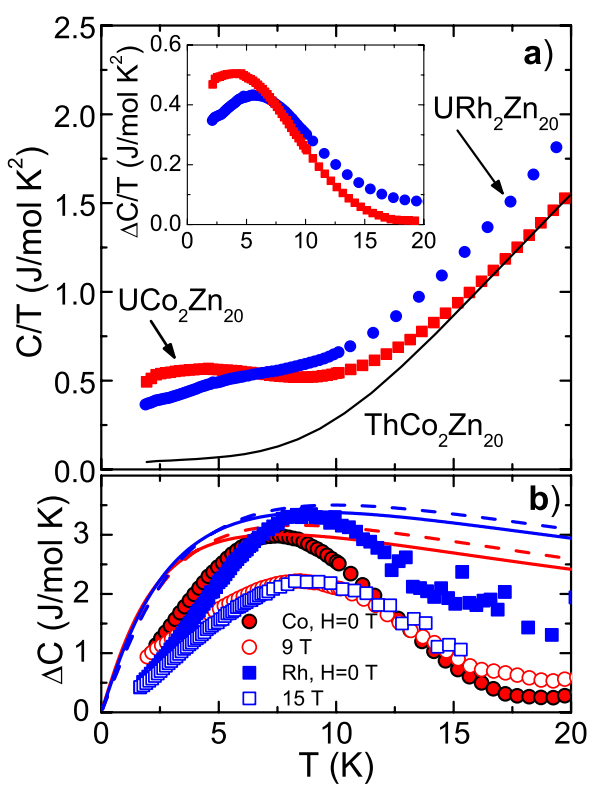

FIG. 3. (Color online) (a) Specific heat $C / T$ vs $T$ of $\mathrm{UCo}_{2} \mathrm{Zn}_{20}$ (red squares) and $\mathrm{URh}_{2} \mathrm{Zn}_{20}$ (blue circles) and for $\mathrm{ThCo}_{2} \mathrm{Zn}_{20}$ (black line) below $20 \mathrm{~K}$. Inset: $5 f$ contribution to the specific heat $\Delta C / T$ vs $T$, for $\mathrm{UCo}_{2} \mathrm{Zn}_{20}$ (red squares) and $\mathrm{URh}_{2} \mathrm{Zn}_{20}$ (blue circles). (b) $5 f$ specific-heat contribution $\Delta C$ vs $T$ of $\mathrm{UCo}_{2} \mathrm{Zn}_{20}$ and $\mathrm{URh}_{2} \mathrm{Zn}_{20}$ in magnetic fields up to $15 \mathrm{~T}$. The solid (zero field) and dashed lines ( $H=9 \mathrm{~T}$ and $15 \mathrm{~T}$ for $\mathrm{M}=\mathrm{Co}$ and $\mathrm{Rh}$, respectively) are fits of the RLM model discussed in the text to the $\Delta C(T)$ data.

where $R$ is the gas constant and $C_{J}$ is the $\mathrm{U} 5 f^{3}$ Curie constant. Using the values at $2 \mathrm{~K}$ for $\chi_{0}$ and $\gamma, \mathcal{R}_{W} \simeq 1.4$ for both compounds.

The momentum-averaged dynamic susceptibility $\chi^{\prime \prime}(\Delta E)$, determined from the scattering function via $S_{\mathrm{mag}}=(n(E)$ +1) $f^{2}(Q) \chi^{\prime \prime}(\Delta E)$, where $f^{2}(Q)$ is the U $5 f$ form factor and $(n(E)+1)$ is the thermal occupation factor, ${ }^{14}$ is plotted vs energy transfer $\Delta E$ in Fig. 4. A fit to an inelastic Lorentzian function $\chi^{\prime \prime}(\Delta E)=\Delta E P(\Delta E)$ with $P(\Delta E)=\chi^{\prime}(0)(\Gamma / \pi) /[(\Delta E$ $\left.\left.-E_{0}\right)^{2}+\Gamma^{2}\right]$, with $E_{0}=3 \mathrm{meV}, \Gamma=5 \mathrm{meV}$, and $\chi^{\prime}(0)=3.3$ $\times 10^{-2} \mathrm{emu} / \mathrm{mol}$, appears to underestimate the data below $\Delta E<5 \mathrm{meV}$, but fits well at higher energy transfer; it slightly underestimates the measured uniform magnetic susceptibility (Fig. 2). A quasielastic scattering function with $E_{0}=0, \Gamma=5 \mathrm{meV}$, and $\chi^{\prime}(0)=5.9 \times 10^{-2} \mathrm{emu} / \mathrm{mol}$ fits well at low energy, but overestimates the data at high-energy transfer and overestimates the susceptibility. The actual line shape is likely intermediate between these two cases and suggests a Kondo energy scale of order $T_{K} \sim 20 \mathrm{~K}$.

Theoretical Anderson impurity model calculations within the $\mathrm{NCA}^{15-17}$ affirm a localized uranium electronic configuration in $\mathrm{UM}_{2} \mathrm{Zn}_{20}(\mathrm{M}=\mathrm{Co}, \mathrm{Rh})$ and provide a realistic crystalline electric-field scheme in (semi-) quantitative agreement with experiment. The proposed model of the $\mathrm{UM}_{2} \mathrm{Zn}_{20}(\mathrm{M}=\mathrm{Co}, \mathrm{Rh})$ compounds is analogous to that applied to Ce-based heavy-fermion compounds in which the localized $4 f^{1}$ configuration is allowed to hybridize with a nonmagnetic $4 f^{0}$ state at the Fermi energy $E_{F}$ to create a Kondo resonance centered at $E_{F}+k_{B} T_{K} \cdot{ }^{15,16}$ In the $\mathrm{UM}_{2} \mathrm{Zn}_{20}(\mathrm{M}=\mathrm{Co}, \mathrm{Rh})$ materials, the two relevant $5 f$ configurations are $5 f^{2}$ and $5 f^{3}$. Over a wide range of cubic CEF

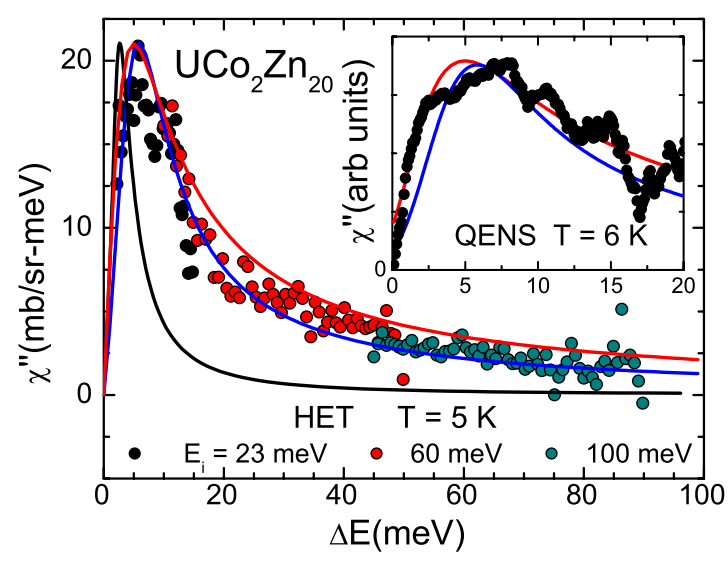

FIG. 4. (Color online) Dynamic susceptibility $\chi^{\prime \prime}$ vs energy transfer $\Delta E$ of $\mathrm{UCo}_{2} \mathrm{Zn}_{20}$. Main panel: HET data at three incident energies at $T=5 \mathrm{~K}$. Inset: data from QENS at $T=6 \mathrm{~K}$. Blue line: inelastic scattering function with $E_{0}=3 \mathrm{meV}, \Gamma=5 \mathrm{meV}, \chi^{\prime}(0)$ $=3.3 \times 10^{-2} \mathrm{emu} / \mathrm{mol}$. Red line: quasielastic scattering function with $\Gamma=5 \mathrm{meV}, \chi^{\prime}(0)=5.9 \times 10^{-2} \mathrm{emu} / \mathrm{mol}$. Black line: AIM prediction as discussed in the text; the overall magnitude has been reduced for comparison with the data.

parameters, the ground state of the $J=4, f^{2}$ configuration is a $\widetilde{\Gamma}_{1}$ singlet; for the same parameters, the ten-fold degenerate $J=9 / 2, f^{3}$ state splits into a doublet $\widetilde{\Gamma}_{6}$ ground state and two excited $\widetilde{\Gamma}_{8}$ quartets. ${ }^{18}$ Only these relevant states are retained and other excited states are neglected. Therefore, the impurity Hamiltonian hybridizes the $f^{2}$ singlet ground state with all states of the $f^{3}$ configuration and can be written as

$$
\begin{aligned}
H= & \sum_{m} \varepsilon_{f m}|m\rangle\langle m|+\sum_{k m} \varepsilon_{k m} c_{k m}^{\dagger} c_{k m}+\sum_{k m}\left(V_{k m}|m\rangle\langle 0| c_{k m}\right. \\
& + \text { H.c. }) .
\end{aligned}
$$

Here $|0\rangle$ represents the $f^{2}$ singlet and $|m\rangle$ corresponds to the state $m$ of the $f^{3}$ configuration. The operator $c_{k m}^{\dagger}$ creates an electron at the conduction band with the same symmetry as that of $|m\rangle$ and wave vector $k=|\mathbf{k}|$. The parameter $\varepsilon_{f m}$ is the energy necessary to take an electron from the Fermi energy and add it to the $f^{2}$ configuration, leaving the state $|m\rangle$. The CEF effects are introduced explicitly through the relation $\varepsilon_{f m}=\varepsilon_{0}+\Delta_{m}$, where $\varepsilon_{0}$ is the energy of the $\widetilde{\Gamma}_{6}$ doublet (taken to be $-2 \mathrm{eV}$ ) and $\Delta_{m}$ are the CEF excitation energies.

Shown as the solid lines in Figs. 2 and 2(a) are fits to the $\chi(T)$ and $M(H)$ data of $\mathrm{UCo}_{2} \mathrm{Zn}_{20}$ by the Anderson impurity model. Excellent quantitative agreement is obtained with the CEF scheme displayed in Fig. 2(b) in which degenerate $\widetilde{\Gamma}_{6}$ and $\widetilde{\Gamma}_{8}$ states are well separated $(\Delta \sim 200 \mathrm{meV})$ from the other $\widetilde{\Gamma}_{8}$ quartet. The fit to $\chi(T)$ reproduces the lowtemperature peak near the Kondo temperature and the roomtemperature susceptibility, but lies slightly above the experimental values at intermediate temperatures, which causes the theoretical $M(H)$ curves for 20 and $40 \mathrm{~K}$ to be slightly larger than the experimental ones [Fig. 2(a)]. The two $\widetilde{\Gamma}_{8}$ quartets are characterized by a mixing parameter $\alpha=-0.997$ of two angular momentum basis states belonging to the $J=9 / 2$ 
multiplet. ${ }^{18}$ The $\widetilde{\Gamma}_{1}$ singlet of the $5 f^{2}$ configuration hybridizes with this quasisextet CEF state with a hybridization strength $\Gamma=97.6 \mathrm{meV}$, corresponding to a $T_{K}=22 \mathrm{~K}$ [defined as the energy difference between the $\widetilde{\Gamma}_{1}$ singlet and $\widetilde{\Gamma}_{6}$ doublet of Eq. (1)] and a $5 f$ occupation number $n_{f}=0.96$, consistent with an extreme Kondo limit in which fluctuations of the $5 f$ charge configuration are suppressed. Similar results are obtained for $\mathrm{URh}_{2} \mathrm{Zn}_{20}$ (Fig. 2) with nearly identical parameters: $\alpha=-0.590, \Gamma=99.1 \mathrm{meV}$, and $T_{K}=26 \mathrm{~K}$. Using these same three parameters $\left(\varepsilon_{0}, \alpha\right.$, and $\left.\Gamma\right)$ that accurately describe the magnetic properties [Figs. 2 and 2(a)] to calculate the dynamic susceptibility (Fig. 4) of $\mathrm{UCo}_{2} \mathrm{Zn}_{20}$, we find that the energy of the peak $(2.64 \mathrm{meV})$ in $\chi^{\prime \prime}(\Delta E)$ in the theory is a factor of two smaller than that seen in the experimental data, and the theoretical width is too small by a similar factor.

Since the peak in specific heat is reminiscent of Kondolike behavior, the resonance level model ${ }^{19}$ (RLM) is used to describe the data, which has been shown to provide a reasonable description of a number of heavy-fermion metals and compares well with more exact treatments. ${ }^{13,20}$ In this model, the physical properties in an applied field are calculated assuming a Lorentzian density of states of width $\delta$ $\left(\sim k_{B} T_{K}\right)$ for arbitrary angular momentum $J$ using the parameters $\delta$, Landé $g$ factor $(=8 / 11)$, and an overall scale factor $a$. As displayed in Fig. 3(b), qualitative agreement is found between the $5 f$ contribution to the specific heat of $\mathrm{UCo}_{2} \mathrm{Zn}_{20}$ and the RLM with the parameters $\delta=9 \mathrm{~K}, J=5 / 2$, and $a$ $=0.8$. Using these same parameters for $\mathrm{UCo}_{2} \mathrm{Zn}_{20}$, the calculated zero-temperature magnetic susceptibility $\chi_{0}$ $=0.031 \mathrm{emu} / \mathrm{mol}$ is in qualitative agreement with the experimental value (Fig. 2); moreover, the Wilson ratio $^{13} \mathcal{R}_{W}$ $=\left(\pi^{2} k_{B}^{2} \chi(0) / \mu_{\text {eff }}^{2} \gamma\right)[1+1 / 2 J]=6 / 5$ is consistent with such a quasisextet CEF state. The field evolution of $\Delta C(T)$ is in rough agreement with the RLM predictions [Fig. 3(b)]; the experimentally observed decrease in magnitude of $\Delta C(T)$ may be a consequence of antiferromagnetic correlations, which would be suppressed in field. The $5 f$ contribution to the entropy at $20 \mathrm{~K}$ is $S_{5 f}(20 \mathrm{~K})=\int^{T}(\Delta C / T) d T$ $\sim 4.0(2) \mathrm{J} / \mathrm{mol} \mathrm{K}$ for $\mathrm{UCo}_{2} \mathrm{Zn}_{20}$, less than the $R \ln (6)$ expected for the quasisextet $\mathrm{CEF}$ state. However, the full entropy of this sixfold degenerate state is only expected to be released below $\sim 80 \mathrm{~K} ;{ }^{13}$ unfortunately, the difference in the phonon spectra between $\mathrm{UCo}_{2} \mathrm{Zn}_{20}$ and $\mathrm{ThCo}_{2} \mathrm{Zn}_{20}$ observed in the neutron scattering spectrum [not shown] precludes a more accurate estimate of $S_{5 f}$ above $\sim 20 \mathrm{~K}$. Similar results are obtained for $\mathrm{URh}_{2} \mathrm{Zn}_{20}$ using the RLM parameters $\delta$ $=11 \mathrm{~K}, J=5 / 2$, and $a=0.9$ [Fig. 3(b)].

It is particularly striking that these $\mathrm{UM}_{2} \mathrm{Zn}_{20}(\mathrm{M}$ $=\mathrm{Co}, \mathrm{Rh})$ compounds can be described in detail as localized heavy-fermion materials. While uranium-based semiconduc- tors, such as $\mathrm{U}_{3} \mathrm{Sb}_{4} \mathrm{Ni}_{3}$, show local-moment behavior, as evidenced by well-defined crystal-field levels in neutron scattering, ${ }^{21}$ this is rarely the case for uranium intermetallic compounds where a featureless inelastic line is typically observed $^{22}$ in the dynamic susceptibility. In the case of $\mathrm{UM}_{2} \mathrm{Zn}_{20}(\mathrm{M}=\mathrm{Co}, \mathrm{Rh})$, the maxima in $\chi(T), C(T)$, and $\chi^{\prime \prime}(\Delta E)$ are well described by the AIM calculations and are consistent with a heavy-fermion state characterized by a Kondo temperature $T_{K} \sim 20-30 \mathrm{~K}$ arising from weak hybridization of conduction-electron states with a localmoment CEF ground state. The fact that virtually identical behavior is observed in the isostructural $\mathrm{YbM}_{2} \mathrm{Zn}_{20}(\mathrm{M}$ $=\mathrm{Fe}, \mathrm{Ru})$ heavy-fermion compounds, ${ }^{23}$ which are unquestionably local-moment systems, reinforces the observation of local-moment behavior in these $\mathrm{UM}_{2} \mathrm{Zn}_{20}(\mathrm{M}=\mathrm{Co}, \mathrm{Rh})$ compounds. Furthermore, similar structural characteristics in $\mathrm{U}_{3} \mathrm{Pd}_{20} \mathrm{Si}_{6}$, whereby both $\mathrm{U}$ atoms in two inequivalent sites are surrounded by cages of $\mathrm{Pd}$ and $\mathrm{Si}$ atoms, presumably lead to a localized uranium configuration as evidenced by the crystal-field excitation at $23 \mathrm{meV}$ (Ref. 24) and localized long-range magnetic order at low temperature. The minimal overlap of the $\mathrm{U}$ ions with neighboring $\mathrm{Zn}$ ligands in these $\mathrm{UM}_{2} \mathrm{Zn}_{20}$ heavy-fermion metals creates an ideal system for thoroughly investigating the Anderson impurity model in actinide compounds.

\section{CONCLUSIONS}

Measurements of magnetic susceptibility, magnetization, specific heat, and dynamic susceptibility of single crystals of $\mathrm{UM}_{2} \mathrm{Zn}_{20}(\mathrm{M}=\mathrm{Co}, \mathrm{Rh})$ are reported. The maximum in the magnetic susceptibility and Sommerfeld coefficient at $T_{\max }$ $\sim 10 \mathrm{~K}$ for both compounds and a broad peak in the dynamic susceptibility at $5 \mathrm{~K}$ in $\mathrm{UCo}_{2} \mathrm{Zn}_{20}$ of width $\Gamma$ $=5 \mathrm{meV}$ suggest a heavy-fermion state characterized by a Kondo temperature $T_{K} \sim 20-30 \mathrm{~K}$ arising from weak hybridization of $f$ - and conduction-electron states due to isolation of the uranium and transition metal atoms in separate $\mathrm{Zn}$ cages. Anderson impurity model fits to the data in the Kondo limit including crystalline electric field effects corroborate an ionic-like uranium electronic configuration in these $\mathrm{UM}_{2} \mathrm{Zn}_{20}$ materials.

\section{ACKNOWLEDGMENTS}

Work at Los Alamos was performed under the auspices of the U.S. DOE. Work at UC Irvine was supported by U.S. DOE Grant No. DE-FG02-03ER46036. S.B. acknowledges the University of Delaware for financial support. V. V. belongs to CONICET (Argentina). A.M.L. and A.A.A. are partially supported by CONICET. This work is sponsored by PICyT 03-12742 and PIP 5254 of CONICET. 
* Present address: University of California, Irvine, Irvine, California 92697, USA

${ }^{1}$ P. Lee, N. Nagaosa, and X.-G. Wen, Rev. Mod. Phys. 78, 17 (2006).

${ }^{2}$ R. A. Borzi, S. A. Grigera, J. Farrell, R. S. Perry, S. J. S. Lister, S. L. Lee, D. A. Tennant, Y. Maeno, and A. P. Mackenzie, Science 315, 214 (2007).

${ }^{3}$ Z. Fisk, H. R. Ott, T. M. Rice, and J. L. Smith, Nature (London) 320, 124 (1986).

${ }^{4}$ H. R. Ott and Z. Fisk, in Handbook on the Physics and Chemistry of the Actinides, edited by A. J. Freeman and G. H. Lander (North-Holland, Amsterdam, 1987), Vol. 5, Chap. 2, p. 85.

${ }^{5}$ G. R. Stewart, Rev. Mod. Phys. 56, 755 (1984).

${ }^{6}$ N. E. Bickers, D. L. Cox, and J. W. Wilkins, Phys. Rev. B 36, 2036 (1987).

${ }^{7}$ A. L. Cornelius, J. M. Lawrence, T. Ebihara, P. S. Riseborough, C. H. Booth, M. F. Hundley, P. G. Pagliuso, J. L. Sarrao, J. D. Thompson, M. H. Jung, A. H. Lacerda, and G. H. Kwei, Phys. Rev. Lett. 88, 117201 (2002).

${ }^{8}$ J. M. Lawrence, P. S. Riseborough, C. H. Booth, J. L. Sarrao, J. D. Thompson, and R. Osborn, Phys. Rev. B 63, 054427 (2001).

${ }^{9}$ E. D. Bauer, J. D. Thompson, J. L. Sarrao, and M. F. Hundley, J. Magn. Magn. Mater. 310, 449 (2007).

${ }^{10}$ P. C. Canfield and Z. Fisk, Philos. Mag. B 65, 1117 (1992).

${ }^{11}$ Further details of the crystal structure investigations can be obtained from the Fachinformationszentrum Karlsruhe, 76344 Eggenstein-Leopoldshafen, Germany, [FAX: (49) 7247-808666; e-mail: crysdata@fiz.karlsruhe.de] on quoting the deposi- tory numbers: CSD $416371\left(\mathrm{UCo}_{2} \mathrm{Zn}_{20}\right)$ and CSD 416369 $\left(\mathrm{URh}_{2} \mathrm{Zn}_{20}\right)$.

${ }^{12}$ V. M. T. Thiede, W. Jeitschko, S. Niemann, and T. Ebel, J. Alloys Compd. 267, 23 (1998).

${ }^{13}$ V. T. Rajan, Phys. Rev. Lett. 51, 308 (1983).

${ }^{14}$ G. L. Squires, Introduction to the Theory of Thermal Neutron Scattering (Dover, New York, 1997).

${ }^{15}$ N. E. Bickers, Rev. Mod. Phys. 59, 845 (1987).

${ }^{16}$ J. E. Han, M. Alouani, and D. L. Cox, Phys. Rev. Lett. 78, 939 (1997).

${ }^{17}$ A. D. Christianson, J. M. Lawrence, A. M. Lobos, A. A. Aligia, E. D. Bauer, N. O. Moreno, C. H. Booth, E. A. Goremychkin, J. L. Sarrao, J. D. Thompson, C. D. Batista, F. R. Trouw, and M. P. Hehlen, Phys. Rev. B 72, 081102(R) (2005).

${ }^{18}$ K. R. Lea, M. J. K. Leask, and W. P. Wolf, J. Phys. Chem. Solids 23, 1381 (1962).

${ }^{19}$ K. D. Schotte and U. Schotte, Phys. Lett. 55A, 38 (1975).

${ }^{20}$ P. Schlottmann, Phys. Rep. 181, 1 (1989).

${ }^{21}$ B. Rainford, D. Adroja, G. Bowden, and I. Benson, Physica B (Amsterdam) 259-261, 417 (1999).

${ }^{22}$ M. Loewenhaupt and C.-K. Loong, Phys. Rev. B 41, 9294 (1990).

${ }^{23}$ M. S. Torikachvili, S. Jia, E. D. Mun, S. T. Hannahs, R. C. Black, W. K. Neils, D. Martien, S. L. Bud'ko, and P. C. Canfield, Proc. Natl. Acad. Sci. U.S.A. 104, 9960 (2007).

${ }^{24}$ N. Tateiwa, N. Metoki, Y. Koike, N. Kimura, H. Aoki, and T. Komatsubara, Physica B (Amsterdam) 312-313, 894 (2002). 